REVIEW Published 14 Jun 2011 | DOI: 10.1038/ncomms1344

\title{
Type la supernovae as stellar endpoints and cosmological tools
}

\author{
D. Andrew Howell1,2
}

Empirically, Type la supernovae are the most useful, precise, and mature tools for determining astronomical distances. Acting as calibrated candles they revealed the presence of dark energy and are being used to measure its properties. However, the nature of the Type la explosion, and the progenitors involved, have remained elusive, even after seven decades of research. But now, new large surveys are bringing about a paradigm shift-we can finally compare samples of hundreds of supernovae to isolate critical variables. As a result of this, and advances in modelling, breakthroughs in understanding all aspects of these supernovae are finally starting to happen.

G uest stars (who could have imagined they were distant stellar explosions?) have been surprising humans for at least 950 years, but probably far longer. They amazed and confounded the likes of Tycho, Kepler and Galileo, to name a few. But it was not until the separation of these events into novae and supernovae by Baade and Zwicky that progress understanding them began in earnest ${ }^{1}$. This process of splitting a diverse group into related subsamples to yield insights into their origin would be repeated again and again over the years, first by Minkowski when he separated supernovae of Type I (no hydrogen in their spectra) from Type II (have hydrogen)' ${ }^{2}$ and then by Elias et al. when they determined that Type Ia supernovae (SNe Ia) were distinct ${ }^{3}$. We now define Type Ia supernovae as those without hydrogen or helium in their spectra, but with strong ionized silicon (SiII), which has observed absorption lines at 6,150,5,800 and 4,000 $($ ref. 4). The progenitor of a Type Ia supernova has never been seen, but the consensus is that they are the result of the thermonuclear explosion of a degenerate carbon-oxygen white dwarf (WD) star ${ }^{5}$ gaining mass in a binary system ${ }^{6}$, in contrast to the other types of supernovae, which are thought to result from the collapse of the core of a massive star. Much about them remains unknown - the process of categorizing and trying to understand them continues.

As distance indicators, $\mathrm{SNe}$ Ia are capable of better than $6 \%$ precision in distance after empirical corrections are made for their lightcurve shape and colour. Just over a decade ago, they were used to deliver perhaps the most shocking scientific discovery in the last half-century: the expansion of the universe is not slowing, but is, instead, accelerating ${ }^{7,8}$. The 'dark energy' required to power this acceleration is prodigious, and, indeed, must be the dominant constituent of the universe by a factor of 17 over normal matter, and 3 over dark matter (which, despite the name, is probably unrelated). But, because dark energy is so dilute $\left(1 \mathrm{~m}^{3}\right.$ contains the energy equivalent to a few atoms of hydrogen), its effects can only be seen on vast scales. We must compare supernovae across $\sim 10 \mathrm{Gyr}$ of cosmic time, during which stars and galaxies have undergone substantial evolution. Therefore, it is critical to understand supernovae, if they are to be relied on as the primary distance probe in astrophysics.

We begin by reviewing SN Ia lightcurves and spectra, and their use in cosmology. This has been refined to the point that SNe Ia are now sensitive to subtle, systematic effects. However, many of these

\footnotetext{
'Las Cumbres Observatory Global Telescope Network, 6740 Cortona Dr., Suite 102, Goleta, California 93117, USA. ${ }^{2}$ Department of Physics, University of California, Santa Barbara, Broida Hall, Mail Code 9530, Santa Barbara, California 93106-9530, USA. Correspondence should be addressed to D.A.H. (email: ahowell@lcogt.net).
} 
are being addressed by comparing large subsamples of hundreds of SNe Ia, which has become possible for the first time only in the last few years.

New multidimensional simulations are revealing once-elusive details of the explosion, such as how asymmetry contributes to the diversity seen in $\mathrm{SNe}$ Ia. Advances in understanding progenitors have been proceeding apace, led by new observations. The properties of $\mathrm{SNe}$ Ia are being correlated with the stellar populations in their host galaxies, yielding real progenitor constraints. Dramatic new discoveries, like supernovae that appear to require a progenitor above the Chandrasekhar mass, are challenging pre-existing theories. Evidence has been mounting that the long-favoured scenario of accretion from a main sequence or red giant companion star cannot account for all $\mathrm{SNe}$ Ia. Instead, the WD merger and even the sub-Chandrasekhar mass explosion models have been experiencing a revival.

Finally, we conclude that empirical SN Ia cosmology remains solid, but is being continually refined by taking into account evermore-subtle effects and new knowledge. But our understanding of the progenitors of $\mathrm{SNe}$ Ia and the explosion process may be undergoing an evolution, as the data from vast new surveys with thousands of supernovae are becoming available.

\section{Lightcurves and spectra}

In a SN Ia, a wave of thermonuclear fusion rips through a degenerate WD star, synthesizing iron-peak elements $(\mathrm{Ni}, \mathrm{Co}, \mathrm{Fe})$ in the dense inner regions, intermediate mass elements $(\mathrm{Si}, \mathrm{S}, \mathrm{Ca}, \mathrm{Mg}$, $\mathrm{O})$ where burning is incomplete, and sometimes leaving unburned material $(\mathrm{C}, \mathrm{O})$ near the outer layers ${ }^{9}$. Though the explosion provides the kinetic energy of the SN Ia and unbinds the WD, this is not what we see as the supernova. The lightcurve is powered by the radioactive decay of ${ }^{56} \mathrm{Ni}$ (half-life 6.1 days) to ${ }^{56} \mathrm{Co}$, and ultimately to ${ }^{56} \mathrm{Fe}$ (half-life 77 days). Gamma rays produced in the decays are thermalized, and at peak light $\sim 85 \%$ of the light output of the SN Ia is in the optical, peaking at $4,000 \AA$, with the remainder mostly radiated in the near-ultraviolet and the near-infrared ${ }^{10}$.

Figure 1 shows the quasi-bolometric (ultraviolet-optical-infrared) lightcurve evolution of a typical SN Ia, SN 2003du (ref. 11), along with representative spectra. SNe Ia rise to maximum light over $\sim 13-23$ days in the $B$-band, with a typical rise time of about 17.5 days $^{12}$. Initially, the time for photons to diffuse out of the dense ejecta is high, so that the rate of deposition of energy by radioactive decay exceeds the energy radiated by the SN. Thus, at early times, spectra show only absorption lines probing the outer layers of the $\mathrm{SN}$. As the SN expands and thins, the light-emitting region (the photosphere) recedes (in mass or velocity coordinates), and spectra start to probe deeper layers. Eventually, the ejecta reach a point where the rate of energy deposition by radioactive decay is equal to the radiated luminosity of the $\mathrm{SN}$, and the $\mathrm{SN}$ starts to decline in luminosity ${ }^{13}$. Around, or after maximum light, spectra show P-Cygni profiles-emission at the rest wavelength (initially weak), and blueshifted absorption. Weeks after maximum light, the spectra start becoming dominated by scattering from permitted lines (at least in the blue) ${ }^{14}$ and ultimately become dominated by emission features as the ejecta start to become optically thin, and the SN makes the transition from the photospheric to the nebular phase. At late times (hundreds of days), gamma rays can freely escape, but positrons may be trapped ${ }^{15}$, and the lightcurve slope may match the slope expected from the decay of ${ }^{56} \mathrm{Co}$, or can be steeper than this in the case of incomplete trapping ${ }^{16}$. Late time spectra are dominated by emission lines from iron-peak elements, synthesized in the deepest regions where the WD was densest.

\section{Cosmology}

Dark energy is often characterized by its ratio of pressure to density, that is, its equation of state, $w=P / \rho c^{2}$ (Fig. 2). If $a$ is an arbitrary length scale in the universe, then the density of some component
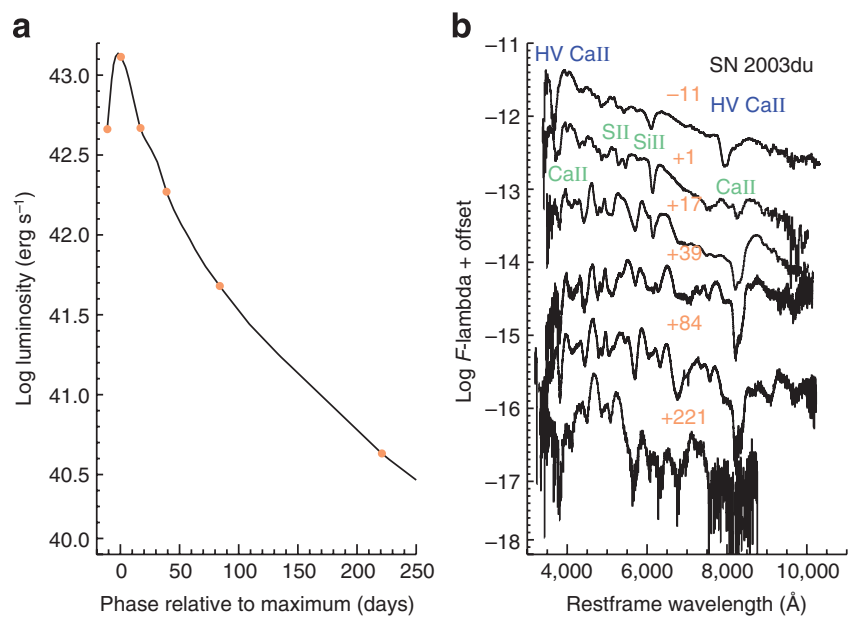

Figure 1 | Bolometric lightcurve and selected spectra from the normal Type la supernova SN 2003du. (a) Quasi-bolometric (ultravioletoptical-infrared) lightcurve for SN 2003du"1. Orange points mark the dates of spectra shown in panel b. (b) Spectra of SN 2003du" ${ }^{11}$, with phases relative to maximum light listed in orange. Characteristic intermediate mass element features seen in maximum light spectra of SNe la are noted in green: Call, SII, and Sill. Before maximum light, when we are seeing the outermost layers of the ejecta, Call is seen at high velocity (HV), a common feature in SNe la. SN ejecta thin with time, and we see to deeper layers. As SNe leave the photospheric phase and enter the nebular phase, emission features start to dominate.

of the universe evolves as $\rho \propto a^{-3(1+w)}$. Normal matter has $w=0$; it dilutes with volume as space expands. If dark energy has an equation of state $w=-1$, corresponding to Einstein's cosmological constant, $\Lambda$, then it has the strange property that the energy density does not dilute as the universe expands; it must be a property of the vacuum. Other possibilities exist: if $w<-1$ the dark energy density is ever growing, and will ultimately result in a 'big rip', destroying galaxies and even subatomic particles. However, $w$ can also be a scalar field with $w>-1$ (generally known as quintessence), and the value of $w$ can evolve over time.

Measurements of $\langle w\rangle$ or $w(z)$ are obtained by building a map of the history of the expansion of the universe using SNe Ia as standardized candles. SNe Ia can show a factor of ten or more difference in peak luminosity, but the luminosity is correlated with the time it takes the supernova to rise and fall in brightness ${ }^{17}$. Therefore, the width of the lightcurve is measured and used to correct the peak luminosity. Common parametrizations include the 'stretch factor', $s$, proportional to lightcurve width, or $\Delta m_{15}(B)$, the number of magnitudes the $B$-band lightcurve falls in 15 days after peak brightness, inversely proportional to lightcurve width ${ }^{17}$. A correction must also be made for colour, since redder SNe are dimmer, both intrinsically, and due to dust ${ }^{18,19}$. Various techniques are used to determine these parameters by fitting lightcurve models to the data, but leading fitters include MLCS2k2 (ref. 20), SALT2 (ref. 21), and SiFTO ${ }^{22}$.

To determine cosmological parameters, an observed Hubble diagram (distance versus redshift) is constructed, and cosmological parameters are varied in a model that is fitted to the data. If we express distances as magnitudes, as a distance estimator, we can $\mathrm{use}^{23}$ :

$$
\mu_{B}=m_{B}^{*}-M+\alpha(s-1)-\beta c,
$$

where $m_{B}^{\star}$ is the peak magnitude of the SN in the $B$-band (the blue filter where the SN is brightest), $s$ is the lightcurve stretch, $c$ is the colour of the SN (a linear combination of $U-B$ and $B-V$, relative to some reference colour $\left.{ }^{22,23}\right), \alpha$ is the slope of the stretch-luminosity relation, $\beta$ is the slope of the colour-luminosity relation, and $M$ is a measure of the absolute magnitude of the SN combined with the 
Hubble constant. Note that since relative magnitudes are used, neither the absolute magnitude of the $\mathrm{SN}$, nor the value of the Hubble constant must be known. $m_{B}^{*}, s$, and $c$ are measured from a fit to each

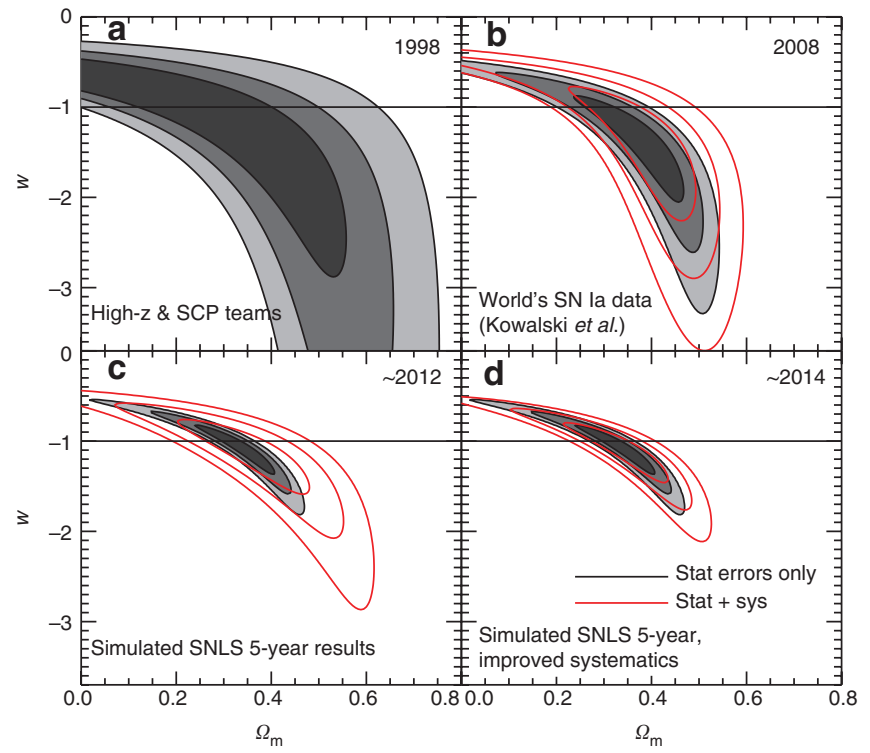

Figure 2 | Improvements in dark energy equation of state constraints from Type la supernovae over time. The top two panels show the remarkable improvements in $w$ made using SNe la over the past decade (assuming a flat universe), the bottom two show the importance of improving systematics in the early years of the next decade ${ }^{111}$. (a) $\Omega_{M}-w$ statistical-only constraints circa $1998^{112}$. (b) By 2008, Kowalski et al., combining many data sets, showed that systematic uncertainties are significant ${ }^{113}$. (c) Expected constraints for the year 5 results of SNLS, assuming additional low- $z \mathrm{SNe}$, and double the number of $z>1 \mathrm{SNe}$ from $\mathrm{HST}$, assuming there is no improvement in systematic uncertainties from the third-year result. (d) Assumes the low-z data are on the Sloan photometric system, and a factor of 2 improvement in measurements of fundamental flux standards. Judged by the area of the inner $68.3 \%$ contour, the improvement from the 1998 results is a factor of 3, 5, and 10 including systematics.
SN lightcurve, while $M, \alpha$, and $\beta$ are constants determined from the overall cosmological fit.

\section{Systematic uncertainties}

Cosmological studies using SNe Ia, have reached a point where over most redshift ranges systematic errors (those that affect many measurements simultaneously in a correlated way) dominate statistical errors (that is, those that are reduced by $\sqrt{N}$ ) ${ }^{24-26}$ - see Figure 2. Many systematic uncertainties can be lowered with new methods, improved statistics, by comparing subsamples of supernovae, or breakthroughs in understanding. The primary systematic uncertainties affecting $\mathrm{SNe}$ Ia are survey-dependent, but there is general consensus that the largest are calibration to the historic Landolt photometric system, treatment of the ultraviolet, reddening due to dust, the differences supernovae show with respect to environment, and the possible evolution of SNe with redshift. See Table 1 for a summary of recent SN Ia constraints on $\langle w\rangle$ and dominant systematic uncertainties.

Calibration. As supernovae are observed at high redshift, the region of the spectrum seen through a given broadband filter changes. A correction must be made for this, the k-correction, which requires knowledge of the spectral energy distribution of an average supernova $^{27}$, and precise knowledge of the filter transmission curves and calibration system used for each supernova. Unfortunately, many historical low-redshift supernovae were transformed to the Landolt photometric standard system, which is poorly understood and no longer reproducible, as the filter and instrument transmission curves used to establish it are not known to modern precision and no longer exist. The fact that the low- $z$ SN sample is on the Landolt system has forced many high redshift surveys to transform to it, incurring a systematic uncertainty in zero points and colour terms ${ }^{23}$. These systematic uncertainties will be reduced once a large new low-redshift sample of $\mathrm{SNe}$ is assembled, calibrated onto a new system (for example, Sloan).

The Ultraviolet. At redshifts above $z \sim 0.2$, the restframe $U$-band and ultraviolet is redshifted into the optical region of the spectrum. Unfortunately, there is a great deal of scatter in restframe $U$ observations. Some surveys rely heavily on the restframe $U$, so uncertainties from this region can translate into dominant systematic uncertainties $^{24}$. In fact, differences seen between the distances determined

Table 1 | Best-fit values of $\langle w\rangle$ and error estimates.

\begin{tabular}{|c|c|c|c|c|}
\hline Systematic & SNLS3 ${ }^{108,109}$ & CfA $^{25} /$ ESSENCE $^{110}$ & SDSS-II24 & SCP26 \\
\hline Best fit w (assuming flatness) & -1.061 & -0.987 & -0.96 & -0.997 \\
\hline Statistical error & - & 0.067 & 0.06 & 0.052 \\
\hline Total stat + systematic error & 0.069 & 0.13 & 0.13 & 0.08 \\
\hline \multicolumn{5}{|l|}{ Systematic error breakdown } \\
\hline Flux reference & 0.053 & 0.02 & 0.02 & 0.042 \\
\hline Experiment zero points & 0.01 & 0.04 & 0.030 & 0.037 \\
\hline Low-z photometry & 0.02 & 0.005 & - & - \\
\hline Landolt bandpasses & 0.01 & - & 0.008 & - \\
\hline Local flows & 0.014 & - & 0.03 & - \\
\hline Experiment bandpasses & 0.01 & - & 0.016 & - \\
\hline Malmquist bias model & 0.01 & 0.02 & - & 0.026 \\
\hline Dust/Colour-luminosity $(\beta)$ & 0.02 & 0.08 & 0.013 & 0.026 \\
\hline SN la Evolution & - & 0.02 & - & - \\
\hline Restframe U band & - & - & 0.104 & 0.010 \\
\hline Contamination & - & - & - & 0.021 \\
\hline Galactic Extinction & - & - & 0.022 & 0.012 \\
\hline
\end{tabular}

For the CfA3/ESSENCE column, $w$ is from Hicken et al. ${ }^{25}$, though uncertainties are from ESSENCE ${ }^{110}$, as they are stated to be similar but are not separately tabulated. The SDSS numbers ${ }^{24}$ are for their SALT2 fit. Errors for each survey use their largest sample. 
by different lightcurve fitters, such as MLCS2k2 (ref. 20), SALT2 (ref. 21), and $\mathrm{SiFTO}^{22}$ boil down largely to the way they treat the restframe $U$-band.

There are two possibilities for $U$-band uncertainty: (1) SNe intrinsically show more variation in $U^{28}$, and/or (2) the $U$-band is poorly calibrated. The Supernova Legacy Survey (SNLS) has shown that when longer-wavelength high-redshift observations from a well-calibrated single survey are k-corrected to restframe $U$-band, the dispersion is more than three times lower than for low-redshift data where the $U$-band is observed directly ${ }^{23}$. Therefore, discrepancies are most likely due to problematic low-redshift observations where many factors conspire to make $U$ observations difficult, including atmospheric variation, extinction, nonstandard filters, and poor calibrators ${ }^{29}$. This systematics can be dealt with by building a new, better-calibrated low-redshift sample, and possibly by avoiding the restframe $U$-band.

Reddening. Lightcurve fitters must correct for the fact that redder supernovae are dimmer. This is due to a combination of an intrinsic colour-luminosity relation (faint supernovae are intrinsically $\operatorname{red}^{18}$ ), and reddening due to dust. Although there is consensus that the two effects ought to be corrected for independently, there is disagreement over whether this can be practically achieved given current data limitations.

MLCS2k2 attempts to separate intrinsic and dust reddening and make different corrections for each ${ }^{20}$. SALT2 and SiFTO work under the assumption that it is difficult or impossible to separate the effects of dust and intrinsic reddening, so they make an empirical reddening correction by solving for the slope of the colour-luminosity relation, $\beta$. If this reddening were only due to dust, then $\beta=R_{\mathrm{B}}=R_{\mathrm{V}}+1$. For Milky Way dust, $R_{\mathrm{B}}=4$.1. However, as shown in Figure 3 , shallower slopes are found for $\beta$. This may result from the conflation of dust and intrinsic SN reddening, and would indicate that the intrinsic SN Ia reddening-luminosity relation has a shallower slope than the dust relation. However, since the lightcurve shape-luminosity relation is already factored out in this method ${ }^{23}$; it means there must be a component of intrinsic colour that does not correlate with lightcurve shape $^{19}$. An alternative is that the dust along the line of sight to SNe Ia is intrinsically different, or that scattering effects in the circumstellar environment result in a different apparent reddening $\operatorname{law}^{30}$.

One way to separate the intrinsic/dust reddening degeneracy is through infrared observations, where the effects of dust are minimized. Infrared observations have shown that many $\mathrm{SNe}$ seem to have a sub-Milky Way value of $R_{\mathrm{v}}$, sometimes as low as $R_{\mathrm{V}}=1.5^{31,32}$. There are hints that lightly reddened $\mathrm{SNe}$ may have an $R_{\mathrm{V}}$ close to that in the Milky Way, whereas heavily extincted SNe Ia have lower values (Fig. 3) 33 . However, this is opposite to earlier findings from optical data ${ }^{34}$, and the small sample size makes it difficult to draw reliable conclusions.

There are indications that supernovae with higher velocity ejecta have lower inferred $R_{\mathrm{V}}$ values $^{35}$ (Fig. 3). As the colour is correlated with physical SN features, this may indicate that sometimes an intrinsic colour difference in $\mathrm{SNe}$ is wrongly ascribed to dust reddening (though this can be mitigated by making a colour cut), or perhaps that there are different progenitor scenarios, with different circumstellar dust-scattering properties, that produce SNe with different velocities.

Various authors have exploited the low sensitivity to dust in the infrared to make infrared Hubble diagrams. This is doubly attractive, because SNe Ia intrinsically show reduced scatter in the infrared $^{36}$-in the $H$-band SNe Ia have an r.m.s dispersion of only 0.15 mag without correction for lightcurve shape or colour ${ }^{37}$. This is theoretically expected, in part because less luminous $\mathrm{SNe}$ are cooler and radiate a larger fraction of their luminosity in the infrared ${ }^{38}$. Although heroic attempts have been made to produce high red-

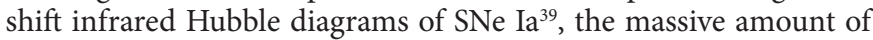
observing time required, and the fact that the restframe infrared

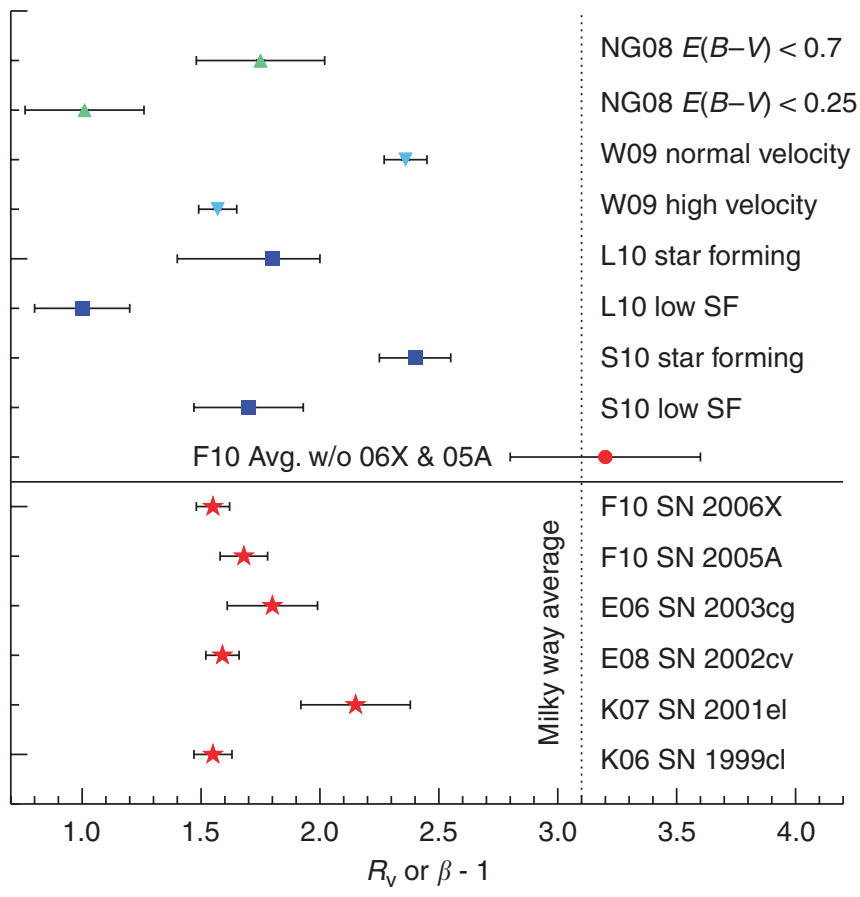

Figure 3 | Determination of the reddening law along the line of site to SNe la. In most cases $R_{V}=A_{V} / E(B-V)$ is shown, but in the S10 data, $\beta-1$ is shown, equivalent to $R_{\vee}$ if the colours of supernovae after lightcurve shape correction are due to dust reddening. Different symbols/colours correspond to different techniques. The most reliable is using $u$ to infrared data to determine the reddening law (red stars), but this can only be done for a few individual SNe la. Data is from K06 (ref. 114), K07 (ref. 32), EO6 (ref. 115), E08 (ref. 31), and F10 (ref. 33). The average from the Carnegie Supernova Program (F10) infrared data, excluding their most reddened events, is shown as a red circle, the only determination consistent with Milky Way dust (dotted line). However, NG08 (ref. 34) (green triangles) come to the opposite conclusion (albeit using only UBVRI data): when they restrict themselves to the least reddened supernovae, they find a lower value of $R_{\mathrm{v}}$. Blue squares show reddening behaviour determined by solving for the reddening law for ensembles of SNe that would minimize their residual on the Hubble diagram. Results split by starforming galaxies and those with low star formation are shown from the SDSS-II SN Survey (L10 (ref. 47)) and the SNLS (S10 (ref. 44)). There are some indications that solving for reddening by minimizing Hubble residuals is incompatible with infrared determinations ${ }^{33}$. Finally, W09 (ref. 35) find that supernovae with high-velocity features in their spectra have a lower $R_{v}$, perhaps an indication that there is a component of reddening that is intrinsic to the SN.

is redshifted to even longer wavelengths, has made serious cosmological constraints from this method elusive.

Populations and evolution. Even before SNe Ia were used for cosmology, it was known that the most luminous SNe Ia (those with the broadest lightcurves), occur only in late-type galaxies ${ }^{40}$. Likewise, subluminous SNe Ia are preferentially found in galaxies with a significant old population ${ }^{41}$. SN lightcurve width and luminosity have now been shown to correlate with host galaxy star formation rate, galaxy mass, and metallicity ${ }^{10,42-44}$.

Because star formation increases by a factor of 10 up to redshift 1.5, and bright, broad-lightcurve supernovae favour star forming hosts, it is expected that the mix of supernovae will change with redshift. Indeed, it has been shown that supernovae at $z=1$ are intrinsically $\sim 12 \%$ more luminous than local SNe Ia ${ }^{45}$. The spectra of high-redshift supernovae also show fewer intermediate mass elements, consistent 


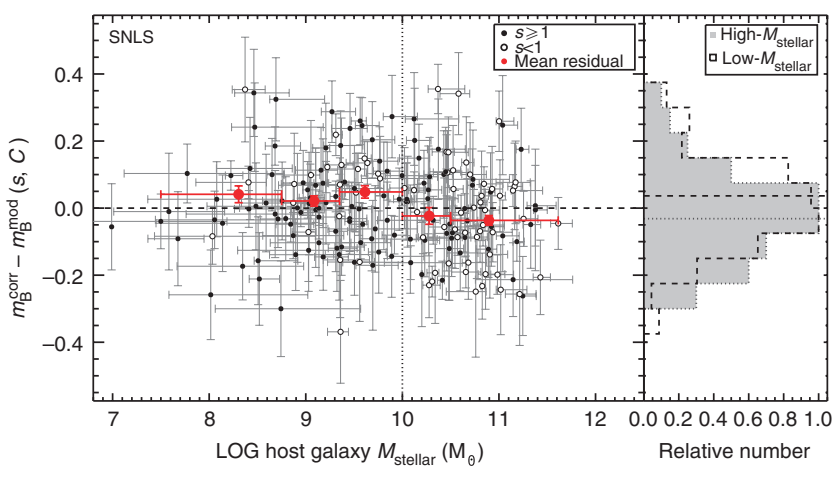

Figure 4 | Residuals from the Hubble diagram for Supernova legacy survey $\mathrm{SNe}$ la as a function of host galaxy mass. Data are from Sullivan et al. ${ }^{44}$ and have been corrected for lightcurve shape and colour. Red points are averages. Histograms on the right show the differences for high- and low-mass host galaxies, split at $M=10^{10} \mathrm{M}_{\odot}$. Horizontal lines show the average residual for each histogram. SNe la in high- and low-mass hosts correct to an absolute magnitude different by $0.08 \pm 0.02 \mathrm{mag}(4 \sigma)$. Supernovae in low-mass galaxies are, on average, brighter than those in high mass galaxies before correction, but are dimmer after s- and c-correction. This galaxy-dependent residual can be corrected by taking host galaxy information into account. Reproduced with permission from John Wiley and Sons.

with the idea that they make more iron-peak elements to power their luminosity ${ }^{46}$. A changing mix of supernovae with redshift is not necessarily problematic for cosmology, if lightcurve shape and colour corrections allow all supernovae to be corrected to the same absolute magnitude. Unfortunately, they do not ${ }^{44,47}$-as is shown in Figure 4, supernovae in high- and low-mass galaxies each correct to an absolute magnitude different by $0.08 \pm 0.02 \mathrm{mag}$. This trend is also present (though weaker), if SNe are split by host star formation rate, or metallicity. If not corrected, this can lead to a $\sim 0.04$ systematic error in $w^{44}$. Therefore, aside from colour and lightcurve shape, a third correction, one for host galaxy properties, must be applied to $\mathrm{SNe}$ Ia to avoid systematic residuals with respect to the Hubble diagram.

The physical origins for the differences in supernova properties in spiral and elliptical galaxies have been elusive ${ }^{40-42}$-are they related to progenitor age, metallicity or entirely different progenitors? The recently discovered trend that galaxy mass may have the most significant role in determining SN properties ${ }^{10,44}$ would seem to implicate metallicity, as higher mass galaxies retain more metals in their deeper potential wells. Still, the age-metallicity degeneracy precludes firm conclusions on this evidence alone ${ }^{48}$. Theoretically, there are reasons that high metallicity progenitors should produce subluminous $\mathrm{SNe} \mathrm{Ia}^{49}$ : increased ${ }^{22} \mathrm{Ne}$ in high metallicity WDs provides more neutrons during the nucleosynthesis that occurs during the explosion, and thus yields more stable ${ }^{58} \mathrm{Ni}$ and less of the radioactive lightcurve-powering ${ }^{56} \mathrm{Ni}$. However, this has only a $\sim 10 \%$ effect on SN luminosity, perhaps indicating that an as-yet unknown metallicity effect or progenitor age is the more important in controlling ${ }^{56} \mathrm{Ni}$ yield and SN luminosity ${ }^{10}$.

If progenitor age is the predominant effect controlling $\mathrm{SN}$ luminosity, this has implications for SN progenitors. In the single degenerate scenario the time to explosion is controlled by the main sequence lifetime of the secondary (mass-transferring) star, as the mass transfer timescale is thought to be negligible, only $\sim 10^{7}$ years. Therefore, in this model, the delay time is a direct indication of the secondary star's mass. In the double degenerate (DD) model, where two WDs merge, gravitational wave radiation ultimately brings the stars together, but they may have a head start if they have drawn closer, by orbiting in the common envelope of one of the stars as it is evolving. Age may still have a role in determining luminosity, because at early times the only WDs will be massive ones derived from the more massive stars.

\section{Explosion theory and observational constraints}

In what might be called the standard model for a SN Ia, a carbonoxygen (CO) WD accretes matter until it compresses to the point that carbon is ignited just before the Chandrasekhar limit (note that the common misconception that the WD goes over the Chandrasekhar limit is wrong - this would lead to collapse to a neutron star). The evidence that the exploding star is a WD is strong, albeit circumstantial: neither hydrogen nor helium is seen in the spectrum of a $\mathrm{SN} \mathrm{Ia}^{50}, \mathrm{SNe} \mathrm{Ia}$ can happen long after star formation has ceased, the explosive process may implicate degenerate matter, the energy obtained by the thermonuclear burning of a WD minus the binding energy roughly matches the kinetic energy of SNe Ia, and simulations of the process have been successful at reproducing SN Ia lightcurves and spectra ${ }^{9,51,52}$.

The WD may have a 'simmering' phase of order a thousand years following unstable carbon ignition, where thermonuclear runaway is prevented by convection ${ }^{53}$. Ultimately, however, explosive burning is ignited and the WD is incinerated in seconds.

If a WD near the Chandrasekhar mass is detonated (that is, the burning occurs supersonically), then the WD burns at such a high density that the fusion products consist almost entirely of iron-peak elements $^{54}$. This does not match the spectra or lightcurves of SNe Ia. A deflagration (subsonic burning), on the other hand, gives the SN time to pre-expand. Burning at a lower density can produce intermediate mass elements and reproduce many of the observational features of $\mathrm{SNe} \mathrm{Ia}^{51}$. However, pure deflagrations fail to produce the high velocity material (Fig. 1) that seems to be nearly ubiquitous in SNe Ia in the outer layers ${ }^{55}$, and in two- and three dimensional simulations they leave too much carbon and oxygen unburned ${ }^{56}$. Therefore, the consensus is that in a Chandrasekhar mass explosion, the flame must start out subsonically, but at some point become supersonic $^{52}$. Though the physics remains poorly understood, models that start as a deflagration but impose a transition to a detonation under certain conditions have been successful at reproducing normal SN Ia lightcurves and spectra, and even lightcurve widthluminosity relations and metallicity effects ${ }^{57}$. Explosions dominated by deflagration produce more intermediate mass elements (dimmer $\mathrm{SNe} \mathrm{Ia}$ ), while those dominated by detonation produce brighter and more ${ }^{56} \mathrm{Ni}$-rich SNe Ia (Fig. 5) ${ }^{57}$.

It is possible to gain insight into the explosion physics with spectropolarimetry or other techniques that reveal asphericity-recent work indicates that some of the dispersion in SN properties results from broken symmetries ${ }^{57}$. SN asymmetry can be measured via spectropolarimetry, since asymmetric electron scattering leads to polarization vectors that do not cancel. Most normal SNe Ia are found to be spherically symmetric ${ }^{58,59}$. The first convincing evidence for significant deviations from spherical symmetry was seen in a subluminous $\mathrm{SN} \mathrm{Ia}^{60}$, which may make sense if they are deflagration-dominated. However, supernovae with high velocity features often show even stronger spectropolarimetric signatures of asymmetry, possibly due to clumpy ejecta ${ }^{58,61}$.

Strangely, SNe Ia whose spectroscopic features start off with high velocity and evolve rapidly, often show nebular lines that appear redshifted, while SNe Ia with slower velocity evolution show blueshifted nebular lines ${ }^{62}$. This probably indicates asymmetry in the explosion, in qualitative agreement with models where a deflagration burns off-center, and is followed by a detonation.

\section{The progenitor question}

Even if there is agreement that the primary star in a SN Ia is a WD, the identity of the secondary star in the binary system is uncertain. There are three broad classes of models: (1) single degenerate ${ }^{6}$, where the companion is a main sequence or red giant star that loses mass via either Roche lobe overflow, or a wind (symbiotic star), (2) 


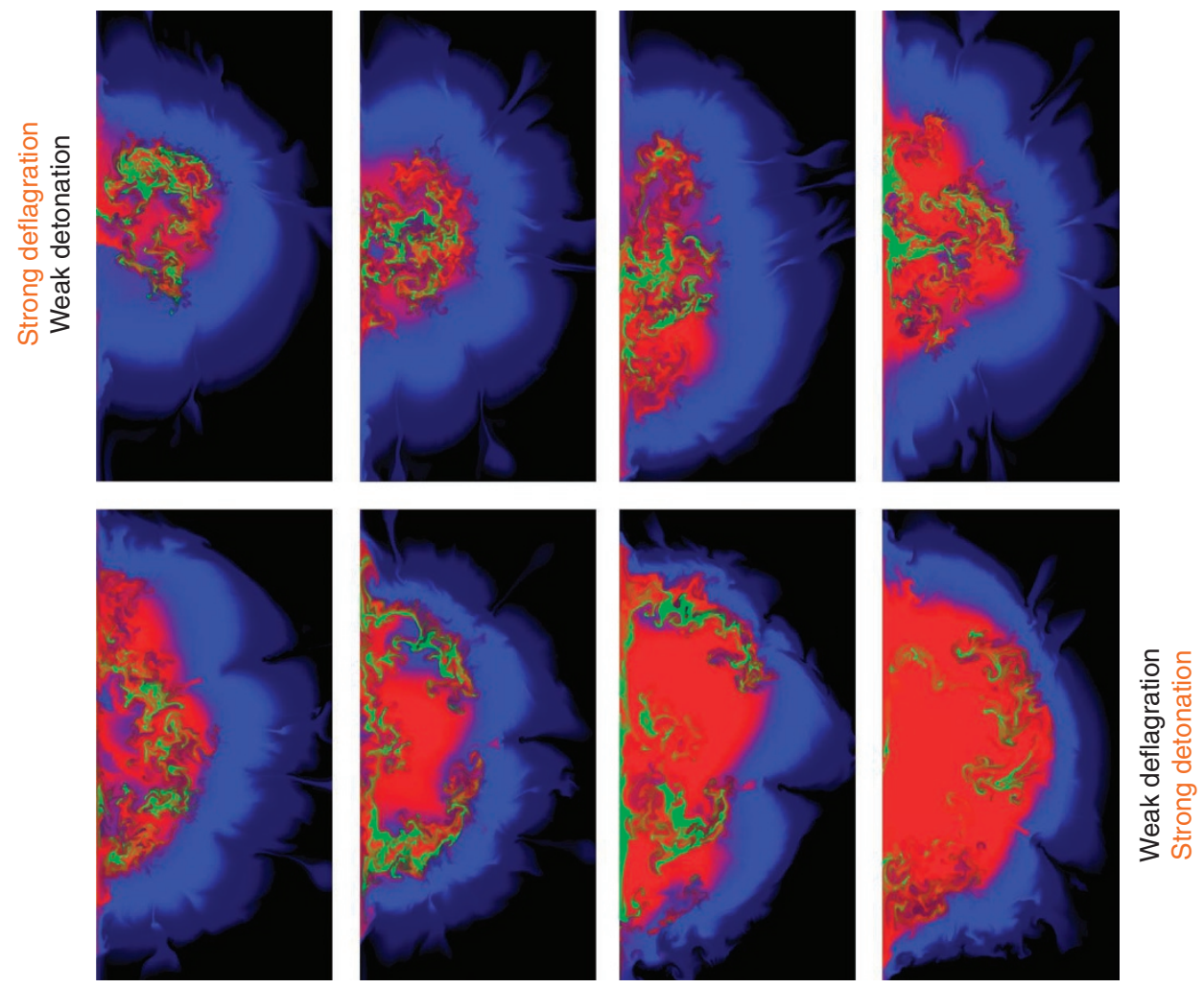

Figure 5 | Two-dimensional SN la models. The simulations, from Kasen et al. ${ }^{57}$, show the relative importance of deflagrations, detonations and asymmetry. Blue shows intermediate mass elements ( $\mathrm{Si}, \mathrm{S}, \mathrm{Ca}$ ), green is stable-iron group elements, and red is ${ }^{56} \mathrm{Ni}$. An initial deflagration wave produces turbulent instabilities, but a later detonation wave burns much of the remaining fuel to ${ }^{56} \mathrm{Ni}$. In low density outer regions the detonation produces only intermediate mass elements. Weak detonations produce the lower ${ }^{56} \mathrm{Ni}$ mass, less luminous $\mathrm{SNe}$ la.

DD, where two WDs merge and explode ${ }^{63,64}$, and (3) sub-Chandra ${ }^{65}$, where a layer of helium builds up on the surface of a WD below the Chandrasekhar mass until it detonates.

Slow accretion. In the single degenerate model, a WD ignites carbon burning, causing it to explode, by accreting matter from a nondegenerate companion to near the Chandra mass. Since CO WDs are thought to start no larger than $\sim 1-1.2$ solar masses, a significant amount of accretion from a secondary star is required. However, hydrogen must be accreted at a rate between $10^{-7}$ and $10^{-8} \mathrm{M}_{\odot} \mathrm{yr}^{-1}$ to steadily burn to carbon or oxygen on the surface of the $\mathrm{WD}^{66}$. If it accretes faster, it will form a red giant-like envelope. Slower accretion is thought to lead to the build up of matter that results in novae, and mass being lost from the system. The apparently required steady burning generates supersoft X-rays, and as a result, systems in this phase of their evolution are observable as supersoft X-ray sources ${ }^{67}$. However, the observed rates of supersoft sources in the Milky Way and external galaxies can only account for $\sim 5 \%$ of the SN Ia rate ${ }^{68,69}$, possibly favouring the DD model, although many of these systems ought to pass through a supersoft phase as well ${ }^{70}$.

White dwarf mergers. In the DD scenario, two WDs merge to achieve the Chandra mass ${ }^{63,64}$. The less massive WD is disrupted into a disk that will eventually accrete onto the more massive $\mathrm{WD}^{71}$. In this case, carbon may be ignited on the surface of the more massive WD during the accretion process, resulting in non-explosive carbon burning, converting the star to an O-Ne WD. This should ultimately undergo accretion induced collapse to a neutron star rather than producing a $\mathrm{SN} \mathrm{Ia}^{72}$, though it may be possible to avoid this fate under certain conditions $^{73}$. The simulated merger of two equal mass $0.9 \mathrm{M}_{\odot}$ WDs has been shown to lead to a SN Ia, although it was subluminous ${ }^{74}$, due to the low central density of a $0.9 \mathrm{M}_{\odot} \mathrm{WD}$. More massive equal-mass mergers may produce normal, or even overluminous SNe Ia, but would be more rare. Lower-mass mergers should be common, though it is not clear if they lead to $\mathrm{SNe} \mathrm{Ia}^{75}$. The merger of two WDs is a very complicated three dimensional process (necessitating approximations in current models), and very few simulations have been completed.

The WD merger scenario has a natural explanation for greater SN luminosity in young environments: younger and more massive stars produce more massive WDs. Massive WD mergers have more potential fuel than less massive mergers.

Sub-Chandrasekhar mass explosions. Although approaching the Chandra mass is a convenient method for triggering carbon burning, there is no hard evidence that the Chandra mass is required. In the classical sub-Chandra models ${ }^{65}$, known as 'double detonation' or 'edge-lit detonations', a layer of accreted helium $\left(\sim 0.2 \mathrm{M}_{\odot}\right)$ is built up either by burning accreted hydrogen to helium or by accretion from a helium-rich donor. When the pressure at the base of the helium layer reaches a critical threshold, it detonates, driving a shock into the core of the WD. This causes a second detonation, resulting in a flame propagating outward from the core (or near it), destroying the WD. Because the sub-Chandra WD has a lower density throughout, a simple detonation does not burn the entire star to iron-peak elements. This model thus has the advantage that an ad hoc deflagration to detonation transition is avoided. However, the significant outer helium layer is efficiently burned to ${ }^{56} \mathrm{Ni}$, resulting in early time spectra that should be rich in ${ }^{56} \mathrm{Ni}$, which does not match observations ${ }^{76}$. If helium can detonate with a smaller layer, which some studies hint ${ }^{77}$, then sub-Chandra simulations can re-

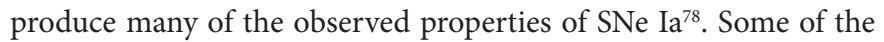
elegance of the model is undercut by the required narrow range of WD masses: $\sim 0.9-1.1 \mathrm{M}_{\odot}$, in order to achieve the central densities necessary to produce iron-peak elements. 
Constraints on progenitors from rates. Different progenitor scenarios lead to different delay times between the birth of the binary system and the explosion as a $\mathrm{SN} \mathrm{Ia}^{79}$. So there is hope of constraining the progenitors by measuring the delay time distribution

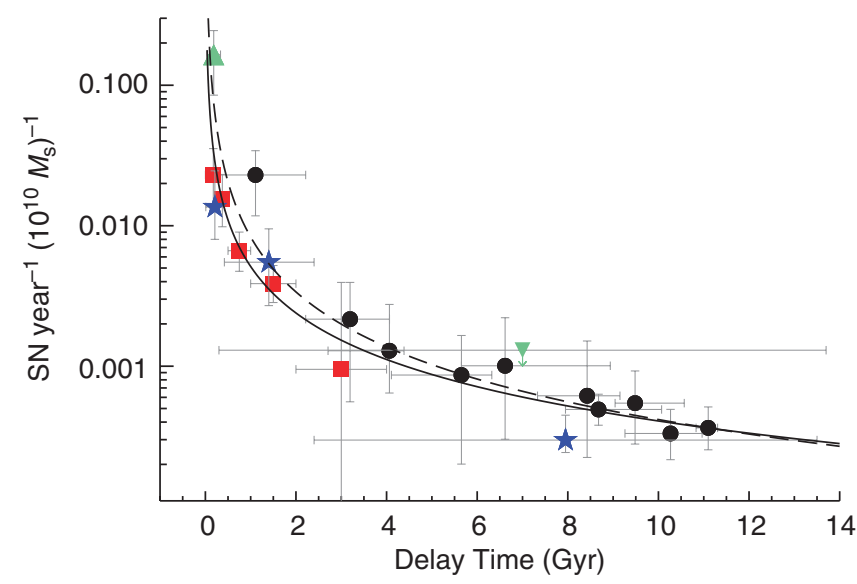

Figure 6 | Delay time distribution for SNe la. Adapted from Maoz, Sharon, and Gal-Yam ${ }^{85}$. All solid circles except the first are delay times derived from cluster $\mathrm{SN}$ rates, assuming formation at $z=3$. The first solid circle results from a constraint on the observed iron-to-stellar mass ratio in clusters. Blue stars are derived from SN rates and galaxy stellar populations in the Lick observatory supernova survey ${ }^{87}$. Green triangles (including an arrow denoting the $95 \%$ confidence upper limit) are constraints from SN remnants in the Magellanic Clouds. Red squares are based on SN la candidates in $\mathrm{E} / \mathrm{SO}$ galaxies at $z=0.4-1.2$ (ref. 84). Curves are power laws $t^{-1.1}$ (solid), and $t^{-1.3}$ (dashed), constrained to pass through the last point. The prediction ${ }^{79}$ from DD mergers is $t^{-1}$. Reproduced by permission of the AAS.
(DTD; Fig. 6). One approach is to measure the lag between the cosmic star formation rate and the SN Ia rate as a function of redshift. However, large uncertainties in each make this particularly difficult, leading authors to different conclusions even when using largely the same data ${ }^{80,81}$.

Another approach is to use the relative $\mathrm{SN}$ rates in different types of galaxies to constrain the DTD. The fact that the SN Ia rate per unit mass is higher by a factor of $\sim 20$ in late-type galaxies indicates that there is a significant population of short-lived thermonuclear $\mathrm{SN}$ progenitors ${ }^{82}$. Whereas there has been much argument about whether the SN Ia DTD is bimodal ${ }^{83}$, a leap forward came with the application of spectral energy distribution fitting techniques to SN host galaxies by modelling their stellar populations ${ }^{42}$. This has allowed more complicated DTDs to be determined: authors using likely SNe Ia from the Subaru-XMM/Newton Deep Survey ${ }^{84}$ (squares in Fig. 6), or in clusters ${ }^{85}$ (filled circles), find a power law DTD proportional to $t^{-1}$. It is difficult to explain the power law DTD results using the single degenerate scenario alone ${ }^{79,84}$, though there is at least one claim ${ }^{86}$.

Host galaxy spectroscopy contains even more information to constrain progenitors. New studies using different methods and different data sets $^{87,88}$, but the same galaxy fitting code, have determined the relative rates of supernovae in $0-0.42 \mathrm{Gyr}$ (prompt), 0.42-2.4 Gyr (medium), and > 2.4 Gyr (delayed) bins (stars Fig. 6). They find that 'prompt' and 'delayed' supernovae are required at several sigma. They also confirm earlier findings that bright, broadlightcurve supernovae favour a prompt population, while dim, narrow-lightcurve SNe Ia favour a delayed population.

Pinpointing the locations of SNe Ia relative to the stellar populations in which they reside can give some information on progenitors, even if it is imprecise due to the probability that the SN has migrated from its birth site. Comparing the distributions of $\mathrm{SNe}$ Ia to the blue light in galaxies reveals that even 'prompt' SNe Ia are

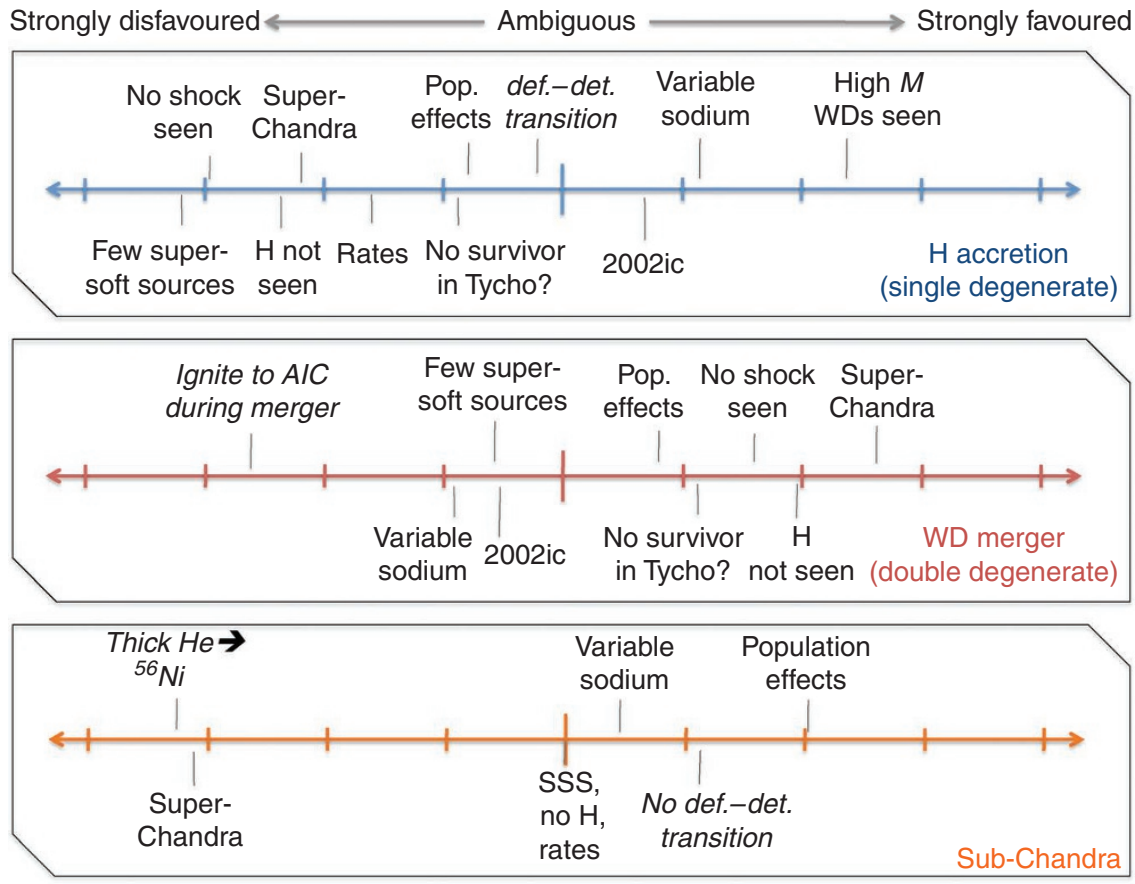

Figure 7 | Schematic illustration of the arguments for and against a given progenitor scenario being the predominant mechanism for SNe la.

Arguments on the left strongly disfavour a certain scenario, whereas arguments on the right strongly favour that model. Note that an argument against a given scenario need not be an equally strong argument for another scenario (that is, the diagram need not be symmetric). Though all evidence is subject to theoretical interpretation, italics indicate exclusively theoretical arguments. Relative rankings reflect an effort to distill community consensus, but are subjective. Arguments and references are described in the text. 'Population effects' refers to the fact that more luminous SNe la are found in younger environments. 'High M WDs seen' refers to the fact that CO WDs $>1.2 \mathrm{M}_{\odot}$ exist in nature, thought to only be possible by slow accretion. 
significantly delayed, with delay times $200-500 \mathrm{Myr}^{89}$. Additional constraints have been obtained by comparing SN Ia remnants in the Magellanic clouds (triangles in Fig. 6) to their local resolved stellar populations ${ }^{90}$. At a $>99 \%$ confidence level, 'prompt' SNe Ia that explode within $330 \mathrm{Myr}$ of star formation are required.

Surviving or preceding material. If WDs grow to become $\mathrm{SNe}$ Ia via hydrogen accretion, then it ought to be possible to see this hydrogen in the SN Ia in some form (Figure 7). However, despite decades of searching, no such smoking gun has been found. No SN Ia has ever been seen in the radio ${ }^{91}$, establishing an upper limit on steady mass loss of material before the SN of $\sim 3 \times 10^{-8} \mathrm{M}_{\odot} \mathrm{yr}^{-1}$. This disfavours the symbiotic star hypothesis, where the WD accretes matter from the stellar wind of its companion. If, on the other hand, the WD is accreting from Roche lobe overflow of a companion, the outer hydrogen layers of the secondary ought to become stripped and entrained in the SN ejecta, where it will show up at low velocities when the ejecta become optically thin ${ }^{92}$. However, in a couple of well-observed SNe Ia, upper limits on the amount of hydrogen detected are $0.01 \mathrm{M}_{\odot}$, which would seem to rule out this scenario ${ }^{50}$, at least for these SNe. In a few cases, apparent SNe Ia have been seen interacting with pre-existing hydrogen, as in $\mathrm{SN}^{93}$, although it is not clear these are really $\mathrm{SNe} \mathrm{Ia}^{94}$, and even if they are, these systems are the exception rather than the rule.

The impact of the SN ejecta on any companion star would shock the ejecta, leaving observational signatures along certain lines of sight for several days, long after the ejecta has overtaken the companion $^{95}$. This would lead to distortions in the early-time lightcurves of SNe Ia around $10 \%$ of the time, especially in the ultraviolet to $B$ regions of the spectrum. If the companion star is a red giant, particularly large distortions ought to be seen-the fact that they would have never seem to be problematic for this scenario ${ }^{96}$.

Variable sodium lines have been seen in high-resolution spectra of some $\mathrm{SNe} \mathrm{Ia}$, and their variability, assumed to be from ionization of circumstellar clouds by the $\mathrm{SN}$, has been taken to be evidence of significant circumstellar material. This ostensibly favours the single degenerate scenario ${ }^{97}$, although maybe only in rare cases-variable sodium was only seen in $6 \%$ of cases in a sample of 31 observations, and then only in the most reddened $\mathrm{SNe} \mathrm{Ia}^{98}$. Not all variable sodium SNe Ia are reddened, however, nor do all reddened supernovae show variable sodium ${ }^{99}$. There have also been doubts that this material is truly circumstellar ${ }^{100}$.

$\mathrm{SN}$ remnants provide more clues to the origin of SNe Ia. They may reveal the progenitor's metallicity ${ }^{101}$, for example. But even more exciting is the prospect that they may house a surviving progenitor star. Tycho's remnant (SN 1572), long suspected of being a SN Ia, has been definitively revealed to be so by modern spectroscopic observations of the SN light, delayed in transit by reflections off of distant interstellar clouds ${ }^{102}$. In the single degenerate scenario, the secondary star should survive the SN explosion, but may be stripped of its outer layers, and heated, such that it takes of order 1,000 years to return to thermal equilibrium ${ }^{92}$. One star, Tycho $G$, has been claimed as evidence for a surviving progenitor ${ }^{103}$, though there are many doubts about this hypothesis. It is too displaced from the centre of the remnant, and its proper motion shows that it did not come from that direction. Also, it was not apparently out of thermal equilibrium, and does not have the expected rotational characteristics ${ }^{104}$.

Super Chandra. Beginning with SNLS-03D3bb ${ }^{105}$, aka SN 2003fg, several supernovae have shown luminosities indicating a nickel yield alone of $1.3-1.8 \mathrm{M}_{\odot}$ (refs 106,107 ), possibly indicating a super-Chandrasekhar mass progenitor. Such energetic explosions ought to have more kinetic energy, and, thus, higher velocity ejecta, but this class of SNe counterintuitively have much slower than average ejecta. This may result from the increased binding energy of a super-Chandra $\mathrm{WD}^{105}$, or from the ejecta plowing into a dense shell of circumstellar material resulting ${ }^{106}$. These $\mathrm{SNe}$ also show rare lines of CII near maximum light, possibly indicating less complete burning. They may also favour a young stellar population. This class of SNe Ia would seem to implicate the merger of two WDs, but no simulation has yet reproduced one.

\section{Final thoughts}

SN Ia are mature probes of cosmology: systematic uncertainties now dominate statistical uncertainties up to $z=1$, and the systematic uncertainties are well characterized. Still, there is plenty of room for improvement in the use of SNe Ia as standard candles.

It is possible that no single model can describe all SNe Ia. Perhaps some SNe Ia are sub-Chandra, some are the result of mergers, and some are caused by Roche Lobe overflow. The explosion of a WD may be similar no matter how it is triggered. If it is not required that a model explain all $\mathrm{SNe}$, this opens the door to scenarios that do not have high expected event rates.

In the study of SNe Ia, we have left the 'serendipity era', when we could only discover what nature happened to tell us through chance encounters with nearby supernovae, and we are entering the 'database era,' where we can proactively ask and answer questions about supernovae by comparing large subsamples. The legacy yields of the multi-year second-generation SN surveys are just becoming apparent. Meanwhile, some third-generation surveys like the Palomar Transient Factory, Pan-STARRS, the Dark Energy Survey, and Skymapper, have already started to produce results, and promise thousands more SNe Ia in the next few years. Finally, fourth-generation surveys, the Large Synoptic Survey Telescope, and possible space missions promise unimaginable riches on the horizon. New techniques like comparing large subsamples, using ultraviolet and infrared data, state of the art calibration, using host galaxy observations, and three-dimensional modelling, have started to yield results, but are still in their infancy. The next decade holds real promise of making serious progress in understanding of nearly every aspect of $\mathrm{SNe}$ Ia, from their explosion physics, to their progenitors, to their use as standard candles. And with this knowledge may come the key to unlocking the darkest secrets of dark energy.

\section{References}

1. Baade, W. \& Zwicky, F. Remarks on super-novae and cosmic rays. Phys. Rev. 46, 76-77 (1934)

2. Minkowski, R. Spectra of supernovae. PASP 53, 224-225 (1941).

3. Elias, J. H., Matthews, K., Neugebauer, G. \& Persson, S. E. Type I supernovae in the infrared and their use as distance indicators. Astrophys. J. 296, 379-389 (1985)

4. Filippenko, A. V. Optical spectra of supernovae. Annu. Rev. Astron. Astrophys. 35, 309-355 (1997).

5. Hoyle, F. \& Fowler, W. A. Nucleosynthesis in supernovae. Astrophys. J. 132, 565-590 (1960).

6. Whelan, J. \& Iben, I. J. Binaries and supernovae of Type I. Astrophys. J. 186, 1007-1014 (1973).

7. Riess, A. G. et al. Observational evidence from supernovae for an accelerating universe and a cosmological constant. Astron. J. 116, 1009-1038 (1998). Co-discoverers of dark energy.

8. Perlmutter, S. et al. Measurements of omega and lambda from 42 high-redshift supernovae. Astrophys. J. 517, 565-586 (1999). Co-discoverers of dark energy.

9. Hillebrandt, W. \& Niemeyer, J. C. Type IA supernova explosion models. Annu. Rev. Astron. Astrophys. 38, 191-230 (2000).

10. Howell, D. A. et al. The effect of progenitor age and metallicity on luminosity and ${ }^{56} \mathrm{Ni}$ yield in Type Ia supernovae. Astrophys. J. 691, 661-671 (2009).

11. Stanishev, V. et al. SN 2003du: 480 days in the life of a normal Type Ia supernova. Astron. Astrophys. 469, 645-661 (2007).

12. Hayden, B. T. et al. The rise and fall of Type Ia supernova light curves in the SDSS-II supernova survey. Astrophys. J. 712, 350-366 (2010).

13. Arnett, W. D. Type I supernovae. I-analytic solutions for the early part of the light curve. Astrophys. J. 253, 785-797 (1982).

14. Branch, D. et al. Comparative direct analysis of Type Ia supernova spectra. IV. Postmaximum. PASP 120, 135-149 (2008).

15. Lair, J. C., Leising, M. D., Milne, P. A. \& Williams, G. G. Late light curves of normal Type Ia supernovae. Astron. J. 132, 2024-2033 (2006).

16. Leloudas, G. et al. The normal Type Ia SN $2003 \mathrm{hv}$ out to very late phases. Astron. Astrophys. 505, 265-279 (2009). 
17. Phillips, M. M. The absolute magnitudes of Type IA supernovae. Astrophys. J. Lett. 413, L105-L108 (1993).

The discovery of the lightcurve shape-luminosity relation.

18. Riess, A. G., Press, W. H. \& Kirshner, R. P. A precise distance indicator: Type IA supernova multicolor light-curve shapes. Astrophys. J. 473, 88 (1996).

19. Conley, A. et al. Is there evidence for a hubble bubble? The nature of Type Ia supernova colors and dust in external galaxies. Astrophys. J. Lett. 664, L13-L16 (2007).

Showed that getting the reddening wrong can have serious consequences for cosmology.

20. Jha, S., Riess, A. G. \& Kirshner, R. P. Improved distances to Type Ia supernovae with multicolor light-curve shapes: MLCS2k2. Astrophys. J. 659, 122-148 (2007).

21. Guy, J. et al. SALT2: using distant supernovae to improve the use of Type Ia supernovae as distance indicators. Astron. Astrophys. 466, 11-21 (2007).

22. Conley, A. et al. SiFTO: an empirical method for fitting SN Ia light curves. Astrophys. J. 681, 482-498 (2008).

23. Astier, P. et al. The supernova legacy survey: measurement of $\Omega_{\mathrm{M}}, \Omega_{\Lambda}$, and $w$ from the first year data set. Astron. Astrophys. 447, 31-48 (2006).

24. Kessler, R. et al. First-year sloan digital sky survey-II supernova results: hubble diagram and cosmological parameters. Astrophys. J. Suppl. Ser. 185, 32-84 (2009).

25. Hicken, M. et al. Improved dark energy constraints from 100 new CfA supernova Type Ia light curves. Astrophys. J. 700, 1097-1140 (2009).

26. Amanullah, R. et al. Spectra and hubble space telescope light curves of six Type Ia supernovae at $0.511<z<1.12$ and the Union2 compilation. Astrophys. J. 716, 712-738 (2010).

27. Hsiao, E. Y. et al. K-corrections and spectral templates of Type Ia supernovae. Astrophys. J. 663, 1187-1200 (2007).

28. Ellis, R. S. et al. Verifying the cosmological utility of Type Ia supernovae: implications of a dispersion in the ultraviolet spectra. Astrophys. J. 674, 51-69 (2008).

29. Jha, S. et al. UBVRI light curves of 44 Type Ia supernovae. Astron. J. 131, 527-554 (2006).

30. Goobar, A. Low $R_{\mathrm{V}}$ from circumstellar dust around supernovae. Astrophys. J. Lett. 686, L103-L106 (2008).

31. Elias-Rosa, N. et al. SN 2002cv: a heavily obscured Type Ia supernova. MNRAS 384, 107-122 (2008).

32. Krisciunas, K. et al. The Type Ia supernova 2004S, a clone of SN 2001el, and the optimal photometric bands for extinction estimation. Astron. J. 133, 58-72 (2007)

33. Folatelli, G. et al. The carnegie supernova project: analysis of the first sample of low-redshift Type-Ia supernovae. Astron. J. 139, 120-144 (2010). Some of the best IR measurements of reddening from the Carnegie Supernova Program.

34. Nobili, S. \& Goobar, A. The colour-lightcurve shape relation of Type Ia supernovae and the reddening law. Astron. Astrophys. 487, 19-31 (2008).

35 . Wang, X. et al. Improved distances to Type Ia supernovae with two spectroscopic subclasses. Astrophys. J. Lett. 699, L139-L143 (2009).

Shows that SNe with different velocities show reddening differences.

36. Krisciunas, K., Phillips, M. M. \& Suntzeff, N. B. Hubble diagrams of Type Ia supernovae in the near-infrared. Astrophys. J. Lett. 602, L81-L84 (2004).

37. Wood-Vasey, W. M. et al. Type Ia supernovae are good standard candles in the near infrared: evidence from PAIRITEL. Astrophys. J. 689, 377-390 (2008).

38. Kasen, D. Secondary maximum in the near-infrared light curves of Type Ia supernovae. Astrophys. J. 649, 939-953 (2006).

39. Freedman, W. L. et al. The carnegie supernova project: first near-infrared hubble diagram to z 0.7. Astrophys. J. 704, 1036-1058 (2009).

40. Hamuy, M. et al. The absolute luminosities of the Calan/Tololo Type IA supernovae. Astron. J. 112, 2391-2397 (1996).

41. Howell, D. A. The progenitors of subluminous Type Ia supernovae. Astrophys. J. Lett. 554, L193-L196 (2001).

42. Sullivan, M. et al. Rates and properties of Type Ia supernovae as a function of mass and star formation in their host galaxies. Astrophys. J. 648, 868-883 (2006).

43. Gallagher, J. S. et al. Supernovae in early-type galaxies: directly connecting age and metallicity with Type Ia luminosity. Astrophys. J. 685, 752-766 (2008).

44. Sullivan, M. et al. The dependence of Type Ia supernovae luminosities on their host galaxies. MNRAS 406, 782-802 (2010).

45. Howell, D. A., Sullivan, M., Conley, A. \& Carlberg, R. Predicted and observed evolution in the mean properties of Type Ia supernovae with redshift. Astrophys. J. Lett. 667, L37-L40 (2007).

46. Sullivan, M. et al. The mean Type Ia supernova spectrum over the past nine gigayears. Astrophys. J. Lett. 693, L76-L80 (2009).

47. Lampeitl, H. et al. The effect of host galaxies on Type Ia supernovae in the SDSS-II supernova survey. Astrophys. J. 722, 566-576 (2010).

48. Gallagher, J. S. et al. Chemistry and star formation in the host galaxies of Type Ia supernovae. Astrophys. J. 634, 210-226 (2005).

49. Timmes, F. X., Brown, E. F. \& Truran, J. W. On variations in the peak luminosity of Type Ia supernovae. Astrophys. J. Lett. 590, L83-L86 (2003).
50. Leonard, D. C. Constraining the Type Ia supernova progenitor: the search for hydrogen in nebular spectra. Astrophys. J. 670, 1275-1282 (2007).

51. Nomoto, K., Thielemann, F.- K. \& Yokoi, K. Accreting white dwarf models of Type I supernovae. III—carbon deflagration supernovae. Astrophys. J. 286, 644-658 (1984).

Presented the W7 model, the most influential model in SN astrophysics.

52. Khokhlov, A. M. Delayed detonation model for type IA supernovae. Astron. Astrophys. 245, 114-128 (1991). Origin of the delayed detonation model.

53. Piro, A. L. \& Chang, P. Convection during the late stages of simmering in Type Ia supernovae. Astrophys. J. 678, 1158-1164 (2008).

54. Arnett, W. D. A possible model of supernovae: detonation of ${ }^{12} \mathrm{C}$. Astrophys. Space Sci. 5, 180-212 (1969).

55. Mazzali, P. A. et al. High-velocity features: a ubiquitous property of Type Ia supernovae. Astrophys. J. Lett. 623, L37-L40 (2005).

56. Gamezo, V. N., Khokhlov, A. M. \& Oran, E. S. Three-dimensional delayeddetonation model of Type Ia supernovae. Astrophys. J. 623, 337-346 (2005).

57. Kasen, D., Röpke, F. K. \& Woosley, S. E. The diversity of Type Ia supernovae from broken symmetries. Nature 460, 869-872 (2009).

58. Leonard, D. C., Li, W., Filippenko, A. V., Foley, R. J. \& Chornock, R. Evidence for spectropolarimetric diversity in Type Ia supernovae. Astrophys. J. 632, 450-475 (2005)

59. Wang, L. \& Wheeler, J. C. Spectropolarimetry of supernovae. Annu. Rev. Astron. Astrophys. 46, 433-474 (2008).

60. Howell, D. A., Höflich, P., Wang, L. \& Wheeler, J. C. Evidence for asphericity in a subluminous Type Ia supernova: spectropolarimetry of SN 1999by. Astrophys. J. 556, 302-321 (2001).

The first convincing evidence for asphericity in a SNe Ia.

61. Kasen, D. et al. Analysis of the flux and polarization spectra of the Type Ia supernova SN 2001el: exploring the geometry of the high-velocity ejecta. Astrophys. J. 593, 788-808 (2003).

62. Maeda, K. et al. An asymmetric explosion as the origin of spectral evolution diversity in Type Ia supernovae. Nature 466, 82-85 (2010).

Showed high velocity features may be a geometric effect resulting from deflagration to detonation transition models.

63. Iben, I. \& Tutukov, A. V. Supernovae of type I as end products of the evolution of binaries with components of moderate initial mass ( $M$ not greater than about 9 solar masses). Astrophys. J. Suppl. Ser. 54, 335-372 (1984).

64. Webbink, R. F. Double white dwarfs as progenitors of R Coronae Borealis stars and Type I supernovae. Astrophys. J. 277, 355-360 (1984).

65. Woosley, S. E. \& Weaver, T. A. The physics of supernova explosions. Annu. Rev. Astron. Astrophys. 24, 205-253 (1986).

66. Nomoto, K. Accreting white dwarf models for type I supernovae. I-Presupernova evolution and triggering mechanisms. Astrophys. J. 253, 798-810 (1982).

67. van den Heuvel, E. P. J., Bhattacharya, D., Nomoto, K. \& Rappaport, S. A. Accreting white dwarf models for CAL 83, CAL 87 and other ultrasoft X-ray sources in the LMC. Astron. Astrophys. 262, 97-105 (1992).

68. Di Stefano, R. The progenitors of Type Ia supernovae. I. Are they supersoft sources? Astrophys. J. 712, 728-733 (2010).

69. Gilfanov, M. \& Bogdán, Á. An upper limit on the contribution of accreting white dwarfs to the typeIa supernova rate. Nature 463, 924-925 (2010).

70. Di Stefano, R. The progenitors of Type Ia Supernovae. II. Are they doubledegenerate binaries? The symbiotic channel. Astrophys. J. 719, 474-482 (2010).

71. Benz, W., Cameron, A. G. W., Press, W. H. \& Bowers, R. L. Dynamic mass exchange in doubly degenerate binaries. I- 0.9 and 1.2 solar mass stars. Astrophys. J. 348, 647-667 (1990).

72. Saio, H. \& Nomoto, K. Evolution of a merging pair of $\mathrm{C}+\mathrm{O}$ white dwarfs to form a single neutron star. Astron. \& Astrophys. 150, L21-L23 (1985).

73. Yoon, S., Podsiadlowski, P. \& Rosswog, S. Remnant evolution after a carbonoxygen white dwarf merger. MNRAS 380, 933-948 (2007).

74. Pakmor, R. et al. Sub-luminous type Ia supernovae from the mergers of equal-mass white dwarfs with mass $0.9 \mathrm{M}_{\odot}$. Nature 463, 61-64 (2010).

75. van Kerkwijk, M. H., Chang, P. \& Justham, S. Sub-Chandrasekhar white dwarf mergers as the progenitors of Type Ia supernovae. Astrophys. J. Lett. 722, L157-L161 (2010). Explores sub-Chandra mergers of white dwarfs leading to SNe Ia.

76. Nugent, P., Baron, E., Branch, D., Fisher, A. \& Hauschildt, P. H. Synthetic spectra of hydrodynamic models of Type IA supernovae. Astrophys. J. 485, 812-819 (1997).

77. Shen, K. J., Kasen, D., Weinberg, N. N., Bildsten, L. \& Scannapieco, E. Thermonuclear. Ia supernovae from helium shell detonations: explosion models and observables. Astrophys. J. 715, 767-774 (2010).

78. Sim, S. A. et al. Detonations in Sub-Chandrasekhar-mass $\mathrm{C}+\mathrm{O}$ white dwarfs. Astrophys. J. Lett. 714, L52-L57 (2010).

79. Greggio, L. The rates of type Ia supernovae. I. Analytical formulations. Astron. Astrophys. 441, 1055-1078 (2005).

80. Strolger, L., Dahlen, T. \& Riess, A. G. Empirical delay-time distributions of Type Ia supernovae from the extended goods/hubble space telescope supernova survey. Astrophys. J. 713, 32-40 (2010). 
81. Kuznetsova, N. et al. A new determination of the high-redshift Type Ia supernova rates with the hubble space telescope advanced camera for surveys. Astrophys. J. 673, 981-998 (2008).

82. Mannucci, F. et al. The supernova rate per unit mass. Astron. Astrophys. 433, 807-814 (2005)

Revival of the idea that a significant population of SNe Ia come from a relatively young population.

83. Mannucci, F., Della Valle, M. \& Panagia, N. Two populations of progenitors for Type Ia supernovae? MNRAS 370, 773-783 (2006).

84. Totani, T., Morokuma, T., Oda, T., Doi, M. \& Yasuda, N. Delay time distribution measurement of Type Ia supernovae by the Subaru/XMM-newton deep survey and implications for the progenitor. PASJ 60, 1327-1346 (2008).

85. Maoz, D., Sharon, K. \& Gal-Yam, A. The supernova delay time distribution in galaxy clusters and implications for Type-Ia progenitors and metal enrichment. Astrophys. J. 722, 1879-1894 (2010)

86. Hachisu, I., Kato, M. \& Nomoto, K. Young and massive binary progenitors of Type Ia supernovae and their circumstellar matter. Astrophys. J. 679, 1390-1404 (2008).

87. Maoz, D. et al. Nearby supernova rates from the lick observatory supernova search. IV. A recovery method for the delay time distribution. MNRAS 412, 1508-1521 (2011).

88. Brandt, T. D. et al. The ages of Type Ia supernova progenitors. Astron. J. 140, 804-816 (2010).

89. Raskin, C., Scannapieco, E., Rhoads, J. \& Della Valle, M. Prompt Ia supernovae are significantly delayed. Astrophys. J. 707, 74-78 (2009).

90. Maoz, D. \& Badenes, C. The supernova rate and delay time distribution in the Magellanic Clouds. MNRAS 407, 1314-1327 (2010).

91. Panagia, N. et al. A search for radio emission from Type Ia supernovae. Astrophys. J. 646, 369-377 (2006)

92. Marietta, E., Burrows, A. \& Fryxell, B. Type IA supernova explosions in binary systems: the impact on the secondary star and its consequences. Astrophys. J. Suppl. 128, 615-650 (2000).

93. Hamuy, M. et al. An asymptotic-giant-branch star in the progenitor system of a type Ia supernova. Nature 424, 651-654 (2003).

94. Benetti, S. et al. Supernova 2002ic: the collapse of a stripped-envelope, massive star in a dense medium? Astrophys. J. Lett. 653, L129-L132 (2006).

95. Kasen, D. Seeing the collision of a supernova with its companion star. Astrophys. J. 708, 1025-1031 (2010).

Shows theoretically that surviving companions should produce observable effects on SN Ia lightcurves.

96. Hayden, B. T. et al. Single or double degenerate progenitors? Searching for shock emission in the SDSS-II Type Ia supernovae. Astrophys. J. 722, 1691-1698 (2010).

97. Patat, F. et al. Detection of circumstellar material in a normal Type Ia supernova. Science 317, 924-926 (2007).

98. Blondin, S. et al. A second case of variable $\mathrm{Na} I \mathrm{D}$ lines in a highly reddened Type Ia supernova. Astrophys. J. 693, 207-215 (2009).

99. Simon, J. D. et al. Variable sodium absorption in a low-extinction Type Ia supernova. Astrophys. J. 702, 1157-1170 (2009).

100. Chugai, N. N. Circumstellar Na I and Ca II absorption lines of type Ia supernovae in the symbiotic scenario. Astron. Lett. 34, 389-396 (2008).
101. Badenes, C., Hughes, J. P., Cassam-Chenaï, G. \& Bravo, E. The persistence of memory, or how the X-ray spectrum of SNR 0509-67.5 reveals the brightness of its parent Type Ia supernova. Astrophys. J. 680, 1149-1157 (2008).

102. Krause, O. et al. Tycho Brahe's 1572 supernova as a standard typeIa as revealed by its light-echo spectrum. Nature 456, 617-619 (2008).

103. Ruiz-Lapuente, P. et al. The binary progenitor of Tycho Brahe's 1572 supernova. Nature 431, 1069-1072 (2004)

104. Kerzendorf, W. E. et al. Subaru high-resolution spectroscopy of star G in the tycho supernova remnant. Astrophys. J. 701, 1665-1672 (2009)

105. Howell, D. A. et al. The type Ia supernova SNLS-03D3bb from a superChandrasekhar-mass white dwarf star. Nature 443, 308-311 (2006) Discovery and theory of super-Chandra SNe Ia.

106. Scalzo, R. A. et al. Nearby supernova factory observations of SN 2007if: first total mass measurement of a Super-Chandrasekhar-mass progenitor. Astrophys. J. 713, 1073-1094 (2010).

107. Taubenberger, S. et al. High luminosity, slow ejecta and persistent carbon lines: SN 2009dc challenges thermonuclear explosion scenarios. MNRAS 412, 2735-2762 (2011)

108. Conley, A. et al. Supernova constraints and systematic uncertainties from the first three years of the supernova legacy survey. Astrophys. J. Suppl. Ser 192, 1-29 (2011).

109. Sullivan, M. et al. SNLS3: constraints on dark energy combining the supernova legacy survey three year data with other probes. Astrophys. J (in the press).

110. Wood-Vasey, W. M. et al. Observational constraints on the nature of dark energy: first cosmological results from the ESSENCE supernova survey. Astrophys. J. 666, 694-715 (2007).

111. Howell, D. A. et al. Type Ia supernova science 2010-2020. ArXiv e-prints 0903.1086 (2009)

112. Garnavich, P. M. et al. Constraints on cosmological models from hubble space telescope observations of high-z supernovae. Astrophys. J. Lett. 493, L53-L57 (1998).

113. Kowalski, M. et al. Improved cosmological constraints from new, old, and combined supernova data sets. Astrophys. J. 686, 749-778 (2008).

114. Krisciunas, K. et al. Photometry of the Type Ia supernovae 1999cc, 1999cl, and 2000cf. Astron. J. 131, 1639-1647 (2006).

115. Elias-Rosa, N. et al. Anomalous extinction behaviour towards the Type Ia SN 2003cg. MNRAS 369, 1880-1900 (2006).

\section{Acknowledgments}

D.A.H. acknowledges support from LCOGT.

\section{Additional information}

Competing financial interests: The author declares no competing financial interests.

Reprints and permission information is available online at http://npg.nature.com/ reprintsandpermissions/

How to cite this article: Howell, D. A. Type Ia supernovae as stellar endpoints and cosmological tools. Nat. Commun. 2:350 doi: 10.1038/ncomms1344 (2011). 